\title{
GLUCK TWISTING 4-MANIFOLDS WITH ODD INTERSECTION FORM
}

\author{
Selman Akbulut and Kouichi Yasui
}

\begin{abstract}
We give a simple criterion when a Gluck twisting an odd smooth 4-manifold along a 2-sphere $S \subset X$ does not change its diffeomorphism type. We obtain this result by handlebody techniques and plug twisting operation, getting a slightly stronger version of the known fact that Gluck twisting of a 2-sphere $S \subset X$ of a compact smooth 4-manifold with an odd spherical class, in the complement of $S$, does not change the diffeomorphism type of $X$. This is the best possible result on Gluck twisting manifolds with odd homology classes.
\end{abstract}

\section{Introduction}

An interesting problem of four-dimensional topology is to determine whether $S^{4}$ has an exotic smooth structure. The Gluck twisting operation [5] has been regarded as a potential method to produce an exotic $S^{4}$ (cf. [7]). This is the operation of removing an embedded $S^{2} \times D^{2}$ from a 4-manifold and regluing it via the non-trivial diffeomorphism of $S^{2} \times S^{1}$. In [1] by Gluck twisting operation, an exotic pair of non-orientable 4-manifolds was constructed. On the other hand, many families of knotted 2-spheres do not change $S^{4}$ by Gluck twisting (e.g. [5, 8, 2, 6, 9]). Indeed, no exotic pair of orientable 4-manifolds, which are related each other by Gluck twist, known to exits. It is thus interesting to see whether Gluck twists can produce an exotic pair of orientable 4-manifolds.

For a smooth 4-manifold $X$ and a smoothly imbedded 2-sphere $S \subset X$ with trivial normal bundle, let $\nu(S) \cong S^{2} \times D^{2}$ denote the tubular neighborhood of $S$. Let $X_{S}^{\circ}$ be the manifold obtained by surgering $X$ along $S$, that is

$$
X_{S}^{\circ}=(X-\nu(S)) \cup D^{3} \times S^{1} .
$$

We call a second homology class $\alpha$ of $X$ is spherical, if $\alpha$ is represented by a continuous map of the 2-sphere. In this paper, by using handlebody techniques and plug twisting operation, we give an alternative proof of the known result (Corollary 1.3). In fact, this approach gives the slightly stronger result below.

Theorem 1.1. Let $X$ be any compact connected smooth 4-manifold, and $S \subset X$ be a smoothly embedded 2-sphere with trivial normal bundle. Then, Gluck twisting $X$ along $S$ does not change the diffeomorphism type of $X$, provided there is a two-dimensional spherical homology class in $X_{S}^{\circ}$ with odd self intersection.

Received by the editors June 22, 2012.

2000 Mathematics Subject Classification. Primary 57R55, Secondary 57R65.

Key words and phrases. 4-manifold; smooth structure; Gluck twist. 
Remark 1.2. Note that this theorem is the best possible result about Gluck twisting odd manifolds. This is because the odd manifold $X=\mathbb{C P}^{2} \# \overline{\mathbb{C P}^{2}}$ contains a 2-sphere $S$ that changes the homeomorphism type by Gluck twisting (consequently $X_{S}^{\circ}$ does not have any odd spherical class). Moreover, [1] implies that one can get an example of a 2 -sphere in an odd non-orientable 4-manifold $Y$ that produces an exotic smooth structure of $Y$ by Gluck twisting. Note that the orientability is not part of the hypothesis of our theorem.

Also note that the condition of having a spherical class in $H_{2}\left(X_{S}^{\circ} ; \mathbb{Z}\right)$ with odd selfintersection is equivalent to having a class of odd self-intersection in the cohomology group $H^{2}\left(X_{S}^{\circ}, \Lambda\right)$ with coefficients, where $\Lambda$ is the group ring $\mathbb{Z}\left[\pi_{1}\left(X_{S}^{\circ}\right)\right]$, since

$$
H^{2}\left(X_{S}^{\circ}, \Lambda\right) \cong H_{c}^{2}\left(\tilde{X}_{S}^{\circ}, \mathbb{Z}\right) \cong H_{2}\left(\tilde{X}_{S}^{\circ}, \mathbb{Z}\right) \cong \pi_{2}\left(X_{S}^{\circ}\right),
$$

where $\tilde{X}_{S}^{\circ}$ denotes the universal cover, and $H_{c}^{2}$ cohomology with compact support.

Corollary 1.3 (cf. [7]). Let $X$ and $S$ be as in Theorem 1.1. If $X-\nu(S)$ has a simply connected codimension 0 submanifold with odd intersection form, then Gluck twisting $X$ along $S$ does not change the diffeomorphism type of $X$.

\section{Gluck twist}

In this paper, we will use the dotted circle notation of 1-handles. Now let us recall the definition of the Gluck twisting operation: let $\varphi: S^{2} \times S^{1} \rightarrow S^{2} \times S^{1}$ be the self-diffeomorphism defined by $\varphi(x, \theta)=\left(\rho_{\theta}(x), \theta\right)$, where $\rho_{\theta}$ denotes the $\theta$ rotation of the unit 2-sphere about the axis through its poles.

Definition 2.1 (Gluck [5]. cf. [7]). Let $X$ be a smooth 4-manifold, and let $S$ be a 2-sphere in $X$ such that its self-intersecion number is zero. Let $X_{S}$ be the smooth 4-manifold obtained from $X$ by removing the regular neighborhood $\nu(S) \cong S^{2} \times D^{2}$ of $S$, and then regluing it via $\varphi$. This construction $X \mapsto X_{S}$ is called the Gluck twisting of $X$ along $S$.

There are several ways to draw a handle diagram of Gluck twists, here we recall the simple method pointed out in [3]. The usefulness of this new method will be evident in the proof of Theorem 1.1

Let $X$ and $S$ be as in Definition 2.1. Consider a handle decomposition

$$
X=\nu(S) \cup \text { handles, }
$$

where we represent $\nu(S) \cong S^{2} \times D^{2}$ by a 0 -framed unknot in the handle diagram. Introduce a canceling 1- and 2-handle pair locally as in the right picture of Figure 1. Surger the introduced $S^{1} \times D^{3}$ to $S^{2} \times D^{2}$, and surger $\nu(S)$ to $S^{1} \times D^{3}$ (i.e. exchange the dot and the 0 as in the bottom picture). Note that this is a plug twist along $\left(W_{-1,0}, f_{-1,0}\right)$ (see [3]). This operation in fact describes the Gluck twist:

Lemma 2.2. The resulting 4-manifold is diffeomorphic to the Gluck twist $X_{S}$. If $X$ has boundary, then we may assume that the diffeomorphism is the identity on the boundary.

Proof. One can easily see that the above operation is removing $\nu(S) \cong S^{2} \times D^{2}$ and regluing it via a self-diffeomorphism (say $\psi$ ) of $S^{2} \times S^{1}$. A theorem of Gluck [5] (cf. [4]) on the diffeotopy group of $S^{2} \times S^{1}$ implies that, we only need to check $\psi$ does not 


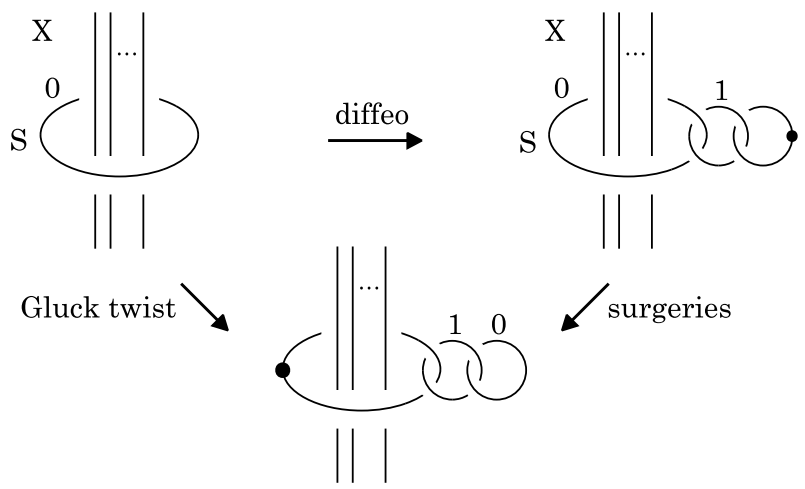

Figure 1. Gluck twist to $X$ along $S$.

extend to a self-homeomorphism of $S^{2} \times D^{2}$. This can be seen by applying the above procedure to the standard handle decomposition of $S^{2} \times S^{2}$ and $S^{2} \times 0$ : the resulting manifold $\mathbb{C P}^{2} \# \overline{\mathbb{C P}^{2}}$ is not homeomorphic to $S^{2} \times S^{2}$.

Remark 2.3. We can similarly prove that the Gluck twist is obtained by the plug twist along $\left(W_{2 m+1,0}, f_{2 m+1,0}\right)$ for any integer $m$.

\section{Proof of the theorem}

For a four-dimensional handlebody $Z$, we denote by $Z^{(i)}$ the subhandlebody of $Z$ consisting of its handles with their indices $\leq i$. We first prepare the lemma below.

Lemma 3.1. Let $Z$ be a smooth compact connected four-dimensional handlebody, and let $f: S^{2} \rightarrow Z$ be a continuous map. We denote by $[f]$ the second homology class of $Z$ given by $f$. Suppose $[f] \neq 0$. Then, if necessary after introducing canceling 2- and 3-handle pairs and sliding 2-handles, $[f]$ is represented by a 2-handle of $Z$ whose attaching circle is null-homotopic in $Z^{(1)}$.

Proof. We may assume that $f$ is an immersion by homotopy. Furthermore, we may assume that the image of $f$ is contained in $Z^{(2)}$, since the cocore of a 3-handle is codimension 3. Let $p_{1}, p_{2}, \ldots, p_{n}$ be the transverse intersection points of $f\left(S^{2}\right)$ and the cocores of 2-handles of $Z$. Let $D_{i}(1 \leq i \leq n)$ be a small 2-disc neighborhoods of $f^{-1}\left(p_{i}\right)$ in $S^{2}$. We may assume that each $f\left(D_{i}\right)$ is a core of a 2-handle of $Z$ and that $f\left(S^{2}-\coprod_{i=1}^{n} D_{i}\right)$ is contained in $Z^{(1)}$. For each $i$, take an $\operatorname{arc} \gamma_{i} \subset S^{2}$ connecting $D_{i}$ and $D_{i+1}$, with $\nu\left(\gamma_{i}\right)$ its tubular neighborhood, so that $\Sigma:=\left(\cup_{i=1}^{n} D_{i}\right) \cup\left(\cup_{i=1}^{n-1} \nu\left(\gamma_{i}\right)\right)$ is a 2-disc in $S^{2}$ and that each $\left.f\right|_{\nu\left(\gamma_{i}\right)}$ is an embedding. We can isotope $f$, so that each $f\left(\nu\left(\gamma_{i}\right)\right)$ is contained in $\partial Z^{(1)}$. Now we obtain a 2-handle of $Z$ whose attaching circle is $f(\partial \Sigma)$, by introducing canceling 2- and 3-handle pairs and sliding 2-handles along the band $f\left(\nu\left(\gamma_{i}\right)\right)$ 's. This 2-handle represents the class $[f]$. Since $S^{2}-\Sigma$ is a 2-disc, the attaching circle $f(\partial \Sigma)=f\left(\partial\left(S^{2}-\Sigma\right)\right)$ bounds an immersed disc in $Z^{(1)}$. Hence, this attaching circle is null-homotopic in $Z^{(1)}$.

We next prove the main theorem. Let $K$ be the attaching circle of the handle representing the spherical homology class of $X_{S}^{\circ}$ with odd self intersection. According to the above lemma, we can assume $K$ is a null homotopic loop in the codimension zero 


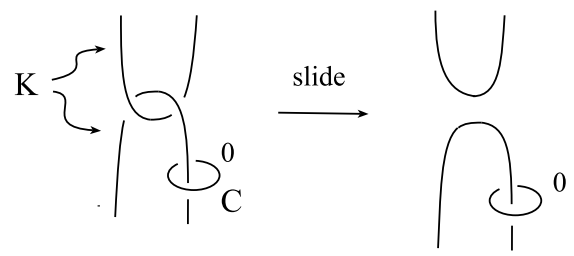

FiguRE 2.

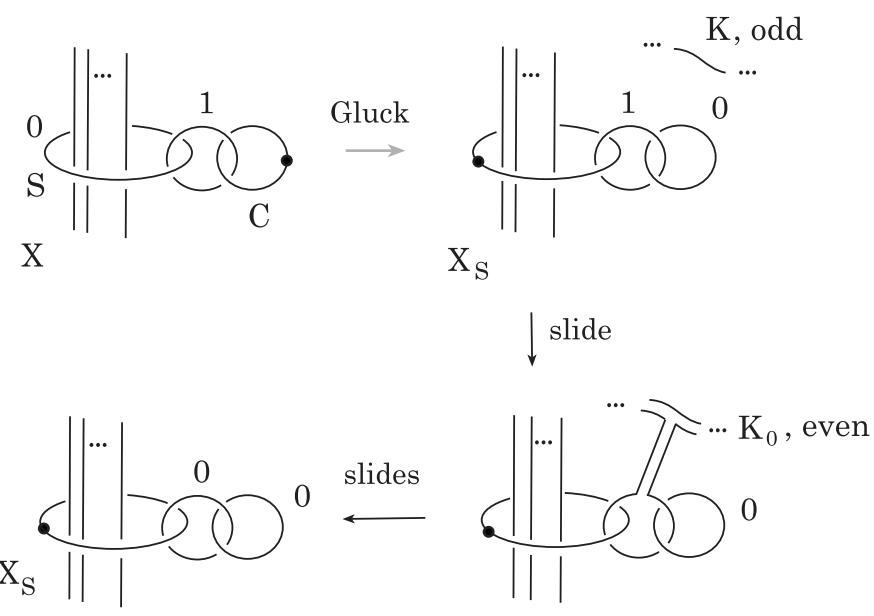

Figure 3. Gluck twist to $X$ along $S$.

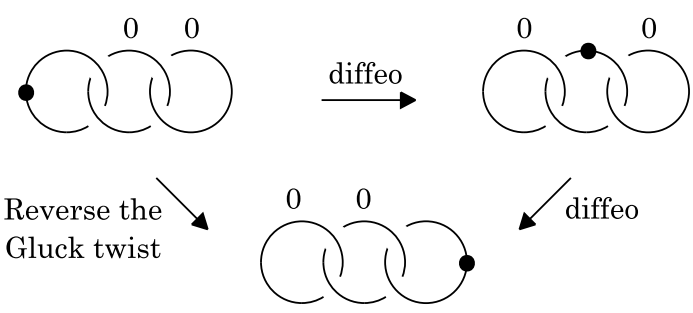

Figure 4. Reversing the Gluck twist to $X$ along $S$.

submanifold $Y$ of $X_{S}^{\circ}$ consisting of its 0- and 1-handles. Hence, after (self crossing) clasp moves $K$ can be made to be an unknot. In particular, if there is another handle represented by a small circle $C$ linking $K$ with zero framing, then by sliding $K$ over $C$ we can make $K$ an unknotted circle as shown in Figure 2.

Having this observation in mind, let us perform the Gluck twisting operation to $X$ along $S$ as shown in Figure 3. Now by sliding the middle 1-framed handle over $K$, we get a 2-handle $K_{0}$ with even framing. By using the above observation, we can isotope $K_{0}$ to the standard middle 0 -framed circle of the last picture of Figure 3.

Here consider the subhandlebody of $X_{S}$ described in the upper left diagram of Figure 4 . We reverse the Gluck twisting operation applied to $X_{S}$. Lemma 2.2 shows that this reversing operation is equivalent to exchanging 0 and the dot as shown 
in the left procedure of Figure 4 . The right procedure of Figure 4 shows that this is a composition of undone operations (recall that every self-diffeomorphism of $\partial D^{4}$ extends to a self-diffeomorphism of $D^{4}$.). Hence $X_{S}$ is diffeomorphic to $X$.

\section{Acknowledgements}

This work was done during the second author's stay at Michigan State University in March 2012 and March 2013. He would like to thank their hospitality. The authors would like to thank Robert Gompf for pointing out an error in an earlier draft of this paper. The first named author is partially supported by the NSF grant DMS 0905917, and the second named author was partially supported by KAKENHI 23840027.

\section{References}

[1] S. Akbulut, Constructing a fake 4-manifold by Gluck construction to a standard 4-manifold, Topology 27(2) (1988), 239-243.

[2] S. Akbulut, Cappell-Shaneson homotopy spheres are standard, Ann. Math. 171 (2010) $2171-2175$.

[3] S. Akbulut and K. Yasui, Corks, Plugs and exotic structures, J. Gökova Geom. Topol., 2 (2008), 40-82.

[4] F. Ding and H. Geiges, The diffeotopy group of $S^{1} \times S^{2}$ via contact topology, Compos. Math. 146(4) (2010), 1096-1112.

[5] H. Gluck, The embedding of two-spheres in the four-sphere, Trans. Amer. Math. Soc. 104 (1962), $308-333$.

[6] R. Gompf, More Cappell-Shaneson spheres are standard, Algebr. Geom. Topol. 10(3) (2010), 1665-1681.

[7] R.E. Gompf and A.I. Stipsicz, 4-manifolds and Kirby calculus, Graduate Studies in Mathematics, 20. American Mathematical Society, 1999.

[8] C. McA. Gordon, Knots in the 4-sphere, Comment. Math. Helv. 51(4) (1976), 585-596.

[9] D. Nash and A.I. Stipsicz, Gluck twist on a certain family of 2-knots, arXiv:1103.5571.

Department of Mathematics, Michigan State University, E.Lansing, Mi, 48824, USA

E-mail address: akbulut@math.msu.edu

Department of Mathematics, Graduate School of Science, Hiroshima University, 1-3-1

KagamiYama, Higashi-Hiroshima, Hiroshima, 739-8526, Japan

E-mail address: kyasui@hiroshima-u.ac.jp 
